\title{
Influence of the PZT Sensor Array Configuration on Lamb Wave Tomography Imaging with the RAPID Algorithm for Hole and Crack Detection
}

\author{
Songlai Wang ${ }^{1}$, Wanrong $\mathrm{Wu}^{1}$, Yiping Shen ${ }^{2, * \mathbb{D}}, \mathrm{Yi} \mathrm{Liu}^{3}$ and Shuai Jiang ${ }^{2}$ \\ 1 College of Mechanical and Electrical Engineering, Central South University, Changsha 410083, China; \\ qingfeng0259@163.com (S.W.); wwr@csu.edu.cn (W.W.) \\ 2 Hunan Provincial Key Laboratory of Health Maintenance for Mechanical Equipment, Hunan University of \\ Science and Technology, Xiangtan 411201, China; jiangshuai88@vip.163.com \\ 3 Zhuzhou National Innovation Railway Technology Co., Ltd., Zhuzhou 412000, China; liuyi_hust@163.com \\ * Correspondence: yiping1011@163.com
}

Received: 3 December 2019; Accepted: 3 February 2020; Published: 6 February 2020

check for updates

\begin{abstract}
The tomography technique is an effective way to quantitatively evaluate damage from reconstruction imaging in structure health monitoring (SHM). The reconstruction algorithm for the probabilistic inspection of damage (RAPID) algorithm based on the signal difference coefficient (SDC) feature is a promising approach due to its superior performance. This paper focuses on the influence of different patterns of PZT (Lead Zirconate Titanate) sensor array configurations, i.e., the circular, square, and parallel array, on reconstruction image qualities for evaluating hole and crack damage. Variable shape parameters are applied to account for the unequal damage distances of different actuator-sensor pairs. Considering the directionality scattering fields of cracks, the angular scattering pattern of the SDC values are studied by simulation. The SDC variations for different groups of sensing paths at the same actuator are applied to predict the crack orientation. An improved RAPID algorithm is proposed by defining an additional SDC value of 1 in the path along the predicted crack orientation, which is determined by the point of the actuator causing the minimal SDC variation and the center point of the initial reconstruction image of the crack. The results show that the improved RAPID algorithm is effective for the evaluation of crack damage. Reconstruction image qualities with three PZT sensor array configurations for both holes and cracks are compared. The research is significant for selecting the PZT sensor array configuration in SHM.
\end{abstract}

Keywords: RAPID algorithm; PZT sensor array configuration; crack orientation; tomography image quality

\section{Introduction}

Lamb wave-based damage detection in plate-like structures draws increasing attention because Lamb waves can travel over a long distance even in materials with high attenuation and which are highly susceptible to damage along the propagation path [1,2]. Computerized tomography (CT) has been introduced in the Lamb-wave-based damage field and has shown great potential in quantitative damage imaging inside materials $[3,4]$. The superior performance of the reconstruction algorithm for the probabilistic Inspection of damage (RAPID) algorithm has been investigated through comparison with different CT algorithms [5,6]. The RAPID algorithm is based on the signal difference coefficient (SDC) feature by comparing the differences between signals with and without damage. The major advantage of the RAPID algorithm is that Lamb wave diffraction is regarded as the elliptical location probability configuration. 
To improve the reconstruction image quality using the RAPID algorithm, Wang [7] introduced Shannon entropy and digital damage fingerprints to highlight the variation in signals caused by damage. Liu [8] combined the RAPID algorithm with the time reversal technique and proposed a new calculation method for the damage index value. Sheen [9] provided a variable shape parameter $\beta$ to account for the unequal damage distances of each sensing path and used this parameter to reconstruct images with multiple defects [10]. Zhao [11] defined a weight function to satisfy the nonuniform section plates. Li [12] calculated the shape parameter $\beta$ for different PZT (Lead Zirconate Titanate) sensor setups in a square array configuration to evaluate surface cracks with irregular shapes in polymer-bonded explosives.

Such improvements of the above developed tomographic techniques focus on the reconstruction algorithm. However, the pattern of the PZT sensor array configuration has not been sufficiently characterized. Croxford [13] recommended a cell of six sensors arranged as a regular hexagon to optimize a practical structure health monitoring (SHM) system. Hall and Michaels [14] explored the sensor number and the pattern of a PZT sensor array on the image performance by a conventional delay-and-sum imaging algorithm. Chen [15] proposed a scattering matrix estimation method to quantify the ability of a PZT sensor array to interrogate a specific directional scatter. Zhao [6] compared the theoretical resolutions in a reconstruction image with that of circular, square, and parallel linear PZT sensor arrays. Wu [16] placed PZT sensors in a square pattern on a stiffened composite panel, and compared the performance with related sensing paths enclosed in a circle and square area. Dziendzikowski [17] defined a signature function for an inhomogeneous noncircular PZT sensor array to normalize the intensity map in a reconstruction image. Some papers can be found that are related to the problem of the optimal sensor placement for complex structures [2,18-21]. Predefined objective functions are used to maximize the coverage of the monitored area while minimizing the number of PZT sensors. However, the pattern of the PZT sensor array configuration is not related to the optimal sensor placement problem.

The objective of this paper is to explore the influences of the pattern of the PZT sensor array configuration on image quality for different types of damage, i.e., a hole and a crack. Holes are the simplest type of scattering features in engineering structures, and their effects on Lamb waves have been extensively studied [22]. Cracks are another common type of scattering feature, but the angular scattering behavior of the Lamb wave manifests significant directionality depending on the angle of the incident wave relative to the crack orientation, which is more complicated than the scattering behavior of a hole [23-28]. Such a directionally scattered field poses great difficulty for crack orientation identification, especially for the asymmetric distribution under oblique wave incidence. Many crack orientation estimation methods have been proposed by finding the maximal value of the reflection coefficient for the normal wave incidence, which is based on the Huygens principle and Snell's law that the incident and reflected angles of the wave are equal [29-33]. Considering that almost no scattering occurs along the crack orientation, Wang [34,35] proposed to correct to 1 the SDC of the path with the minimal SDC peak value in a group of sensing paths, in which the actuator aligns with the crack orientation direction, to improve the tomographic image quality. A similar SDC value modification is introduced after the crack orientation is evaluated based on the SDC variations under different incident directions in this paper.

This paper refers to three patterns of PZT sensor array configuration, e.g., the circular, square, and parallel linear arrays. The RAPID algorithm is utilized to reconstruct images of both a hole and a crack, and variable shape parameters are applied to account for the unequal damage distances. Finite element analysis (FEA) is performed to acquire the complex angular scattering pattern of the SDC value. Considering that the cracks are sufficiently narrow, almost no scattering occurs along the crack orientation when the incident wave is parallel to the crack orientation. The crack orientation is estimated by finding the actuator causing the minimal SDC variation in all groups of sensing path. An improved RAPID algorithm is proposed by defining an additional SDC value of 1 in the path along the predicted crack orientation. The effectiveness of the proposed method is verified. Reconstruction 
image qualities with three patterns of PZT sensor array configurations for both hole and crack are compared. The results are significant for selecting the PZT sensor array configuration in SHM.

\section{The RAPID Algorithm}

The RAPID algorithm is a probabilistic method for reconstructing damage images by comparing the differences between the signals scattered with damage and the reference signals without damage. The signal comparison is based on a damage index known as the SDC. It is very sensitive to the subtle change of the transmitted signal through a damage [6]. The first step of the RAPID algorithm is to calculate the SDC value for all actuator-sensor pairs. The first arrival waves are used to calculate the SDC, which means that the RAPID algorithm is effective only when the damage is inside the sensor array coverage area. The calculation of the SDC between the signal $x_{t r}$ and the reference signal $y_{t r}$ is expressed as [9]

$$
\mathrm{SDC}_{t r}=1-\left|\frac{\sum_{k=1}^{k}\left(x_{t r}-\mu_{x}\right)\left(y_{t r}-\mu_{y}\right)}{\sqrt{\sum_{k=1}^{k}\left(x_{t r}-\mu_{x}\right)^{2}} \sqrt{\sum_{k=1}^{k}\left(y_{t r}-\mu_{y}\right)^{2}}}\right|
$$

where $\mu_{x}$ and $\mu_{y}$ are the means of the corresponding signals and $k$ is the length of the data. If the two signals are identical, the SDC value is zero. If the two signals are completely out of phase, the SDC value is 1 .

In the RAPID algorithm, the image is generated by spatially distributing each SDC value on the image plane in an elliptical pattern. The two focus of the ellipse are located at the positions of the corresponding actuator and sensor, as shown in Figure 1. The elliptical distribution function is defined as

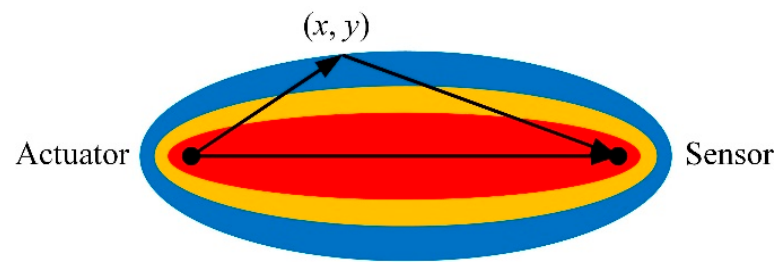

Figure 1. The elliptical distribution function of the reconstruction algorithm for the probabilistic inspection of damage (RAPID).

$$
\begin{cases}S_{t r}(x, y)=\frac{\beta-R_{t r}(x, y)}{1-\beta}, & \text { for } \beta>R_{t r}(x, y) \\ S_{t r}(x, y)=0, & \text { otherwise. }\end{cases}
$$

where the shape parameter $\beta$ controls the size of the ellipse. $R_{t r}(x, y)$ is the ratio of the sum of the distances of the point $(x, y)$ to the sensor point $\left(x_{t}, y_{t}\right)$ and the receiver point $\left(x_{r}, y_{r}\right)$ and is expressed as

$$
R_{t r}(x, y)=\frac{\sqrt{\left(x-x_{t}\right)^{2}+\left(y-y_{t}\right)^{2}}+\sqrt{\left(x-x_{r}\right)^{2}+\left(y-y_{r}\right)^{2}}}{\sqrt{\left(x_{t}-x_{r}\right)^{2}+\left(y_{t}-y_{r}\right)^{2}}}
$$

The traditional algorithm stipulates only that the shape factor $\beta$ is greater than 1.0. For different distances between the actuator and sensor, the variable shape factor is defined as [9]

The improved elliptical distribution function is rewritten as

$$
\begin{cases}S_{t r}(x, y)=\frac{\beta_{t r}-R_{t r}(x, y)}{\beta-1}, & \text { for } \beta_{t r}>R_{t r}(x, y) \\ S_{t r}(x, y)=0, & \text { otherwise. }\end{cases}
$$


The image amplitude at each pixel is the summation of SDC values from each actuator-sensor pair:

$$
P(x, y)=\sum_{t=1}^{N-1} \sum_{r=t+1}^{N} \operatorname{SDC}_{t r} S_{t r}(x, y)
$$

where $N$ is the total number of actuator-sensor pairs.

Considering the small width of the crack damage, the signal transmitted through the crack is almost the same when the sensing path is parallel to the crack length. The angular scattering pattern of SDC value is investigated by FEA, and the criterion to determine the crack orientation is detailed in Section 4. After the crack orientation is determined, the following SDC correction is applied [34]

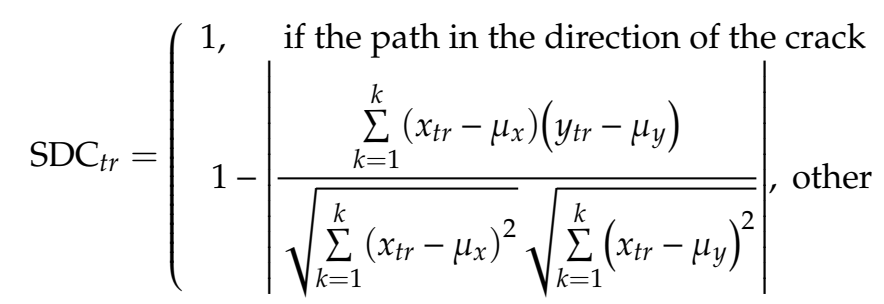

\section{Experimental Setup}

Square aluminum plate specimens with dimensions of $1 \times 1 \mathrm{~m}$ and a thickness of $1 \mathrm{~mm}$ were used. Their density was $2730 \mathrm{~kg} / \mathrm{m}^{3}$, the elastic modulus $68.9 \mathrm{GPa}$, and the Poisson ratio 0.33 . $c_{L}$ and $c_{T}$ were $6240 \mathrm{~m} / \mathrm{s}$ and $3040 \mathrm{~m} / \mathrm{s}$, respectively, the phase and group velocity was plotted in Figure 2 .

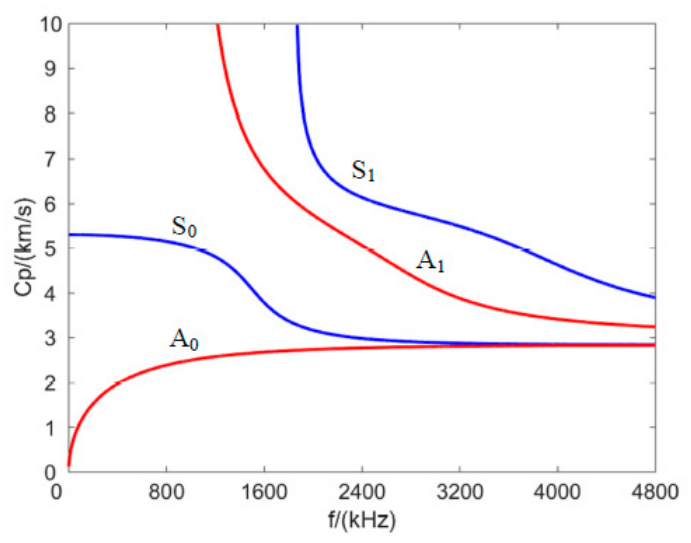

(a)

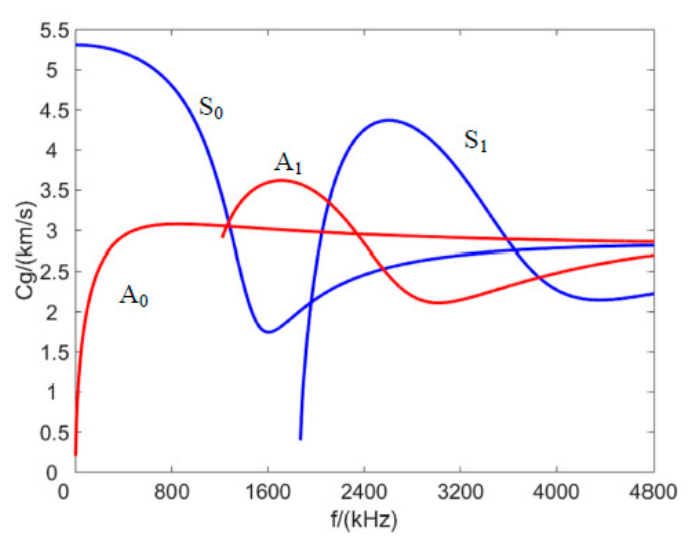

(b)

Figure 2. Disperse curve of (a) phase velocity and (b) group velocity.

Two types of artificial damage were introduced: a hole with a radius of $30 \mathrm{~mm}$ and a crack with a length of $30 \mathrm{~mm}$ and a width of $1 \mathrm{~mm}$. Piezoelectric wafers with a radius of $10 \mathrm{~mm}$ and thickness of $0.3 \mathrm{~mm}$ were used. Sixteen sensors were arranged in circular, square, and parallel linear array configurations. All sensing paths for the three PZT sensor array configurations are shown in Figure 3. Their centers coincide with the center of the specimen. The radius of the circular array and the edge length of the square and the parallel linear array were $350 \mathrm{~mm}$, which were smaller than the dimension of the specimen to ensure infinite conditions of Lamb wave propagation. Variable shape factor values $\beta$ of different sensing paths in three PZT sensor array configurations were calculated. Figure 4 shows the shape factor values $\beta$ related to a hole in the specimen, which are significantly different for different sensing paths in different PZT sensor array configurations. 


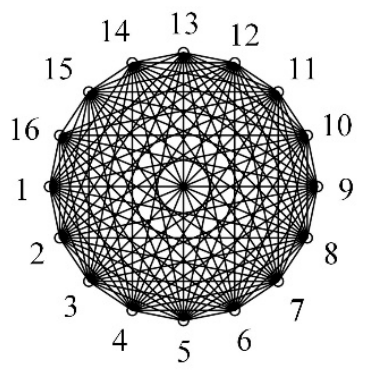

(a) Circular array

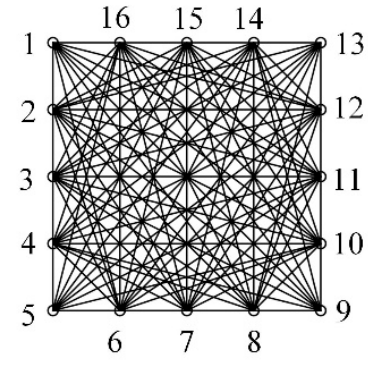

(b) Square array

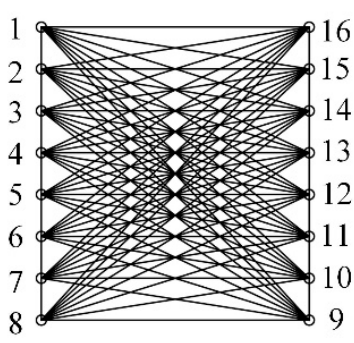

(c) Parallel linear array

Figure 3. Three patterns of PZT (Lead Zirconate Titanate) sensor array configuration and their sensing paths.

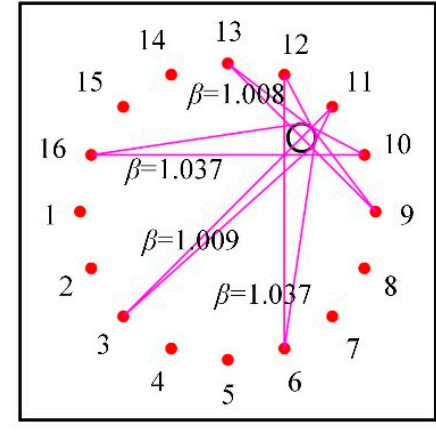

(a) Circular array

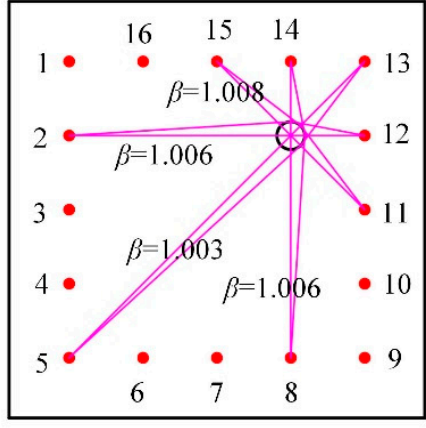

(b) Square array

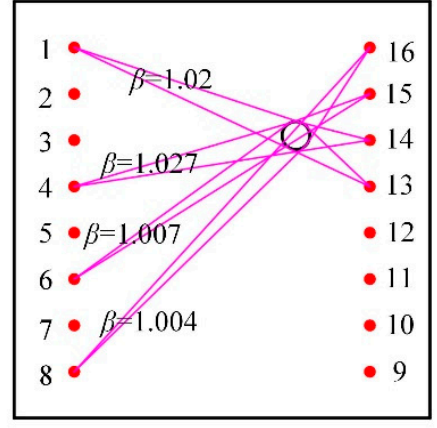

(c) Parallel linear array

Figure 4. Variable shape factors for three PZT sensor array configurations for the specimen with a hole.

A five-cycle narrowband tone-burst signal modulated by the Hamming window was used to excite the Lamb wave in the plate. Lamb waves were generated and measured by using piezoelectric wafers. The central frequency of the excitation wave was $30 \mathrm{kHz}$. $\mathrm{A}_{0}$ mode was excited very strongly at such low frequencies while the $\mathrm{S}_{0}$ mode was barely visible [36,37]. The $\mathrm{A}_{0}$ mode was commonly used for damage detection because of its short wave length and low wave velocity. An EPA-10 power amplifier, produced by Piezo System Inc., (Cambridge, MA, USA,) was used to amplify the excitation narrowband signals. An $80 \mathrm{~V}$ peak-to-peak amplification excitation was applied to the actuator. The data acquisition device NI USB-6366 was applied to collect the signals of PZT sensors. The sampling frequency was $2 \mathrm{MS} / \mathrm{s}$. The experimental test setup is shown in Figure 5a. The scattered Lamb wave signals and the reference signals of all the sensing paths were performed. The signals in the path $A_{3}-S_{11}$ with and without hole damage for the circular PZT array are plotted in Figure $5 b$, where $A_{3}$ denotes that the actuator is the piezoelectric wafer numbered $3, S_{11}$ denotes that the sensor is the piezoelectric wafer numbered 11. The amplitude of $\mathrm{A}_{0}$ mode was larger than the $\mathrm{S}_{0}$ mode, which was recognized based on its group velocity in Figure 2, thus only the $\mathrm{A}_{0}$ wave was used to calculate the SDC. The measured $\mathrm{A}_{0}$ wave amplitudes and phases with and without damage were significantly different. According to Equation (1), SDC values of all sensing paths in three PZT sensor array configurations were calculated based on the measured signals. 


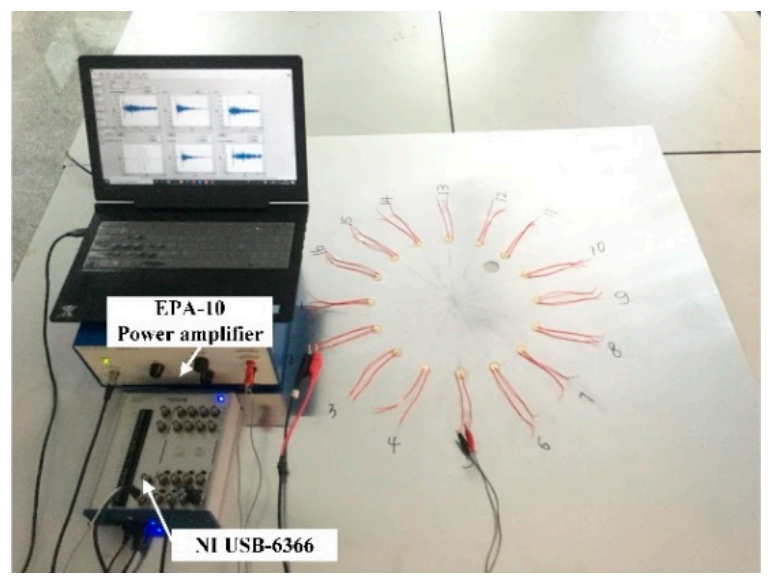

(a)

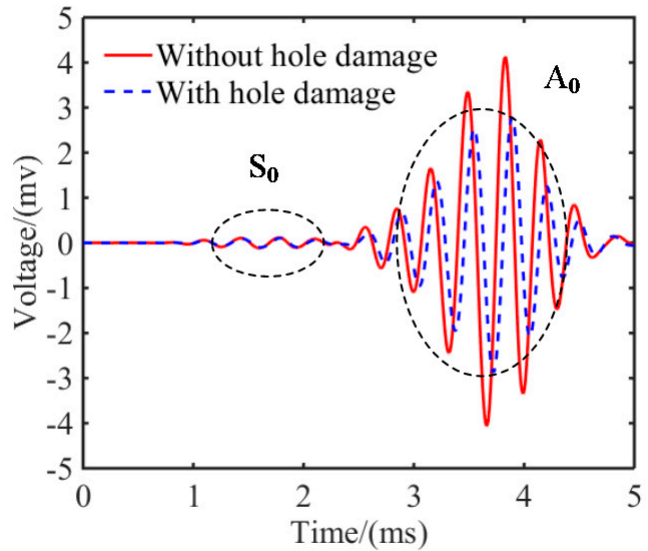

(b)

Figure 5. Experimental test setup and the measured signals in one damage path: (a) experimental test setup and (b) measured signals in the path of $A_{3}-S_{11}$.

\section{Results and Discussion}

\subsection{Influence of PZT Sensor Arrays on the Images of Holes}

The reconstruction images of the hole in the specimen with three patterns of PZT sensor array configurations are shown in Figure 6, and the resultant location and area errors are listed in Table 1, where the damage shape accuracy is the ratio of long to short length of the tomography images after a threshold value. The threshold value was set as 0.7 and applied for the other images in this paper. Note that the use of different patterns of the PZT sensor array configuration has a significant influence on the imaging qualities. The circular array showed the best performance; the location, the area, and the shape were almost the same as for the real hole. The square array had the same location as the real hole but did not have the appropriate hole shape. The parallel linear array had poor accuracy; the location was close to the real hole location, but the elongated shape was quite different from the real shape of the hole. This seems to be related to the total number of the effective sensing paths and its inhomogeneous distribution [6,17], as shown in Figures 3 and 4. The total numbers of the sensing paths was 74 for the circular array and the square array, and 60 for the parallel linear array; the sensing paths and their intersections of the circular array were more homogeneously distributed than the square array, thus the circular array has better performance for shape detection; the elongated shape is caused by a very low density of the parallel linear array.

Table 1. The resultant imaging accuracy of a hole with three PZT sensor array configurations.

\begin{tabular}{ccccc}
\hline $\begin{array}{c}\text { Pattern of the PZT } \\
\text { Sensor Array }\end{array}$ & Real Location & Predicted Location & $\begin{array}{c}\text { Damage Shape } \\
\text { Ratio }\end{array}$ & $\begin{array}{c}\text { Damage Area } \\
\text { Coverage/\% }\end{array}$ \\
\hline Circular & $(255,255)$ & $(257,255)$ & 1.0 & 92.2 \\
Square & $(255,255)$ & $(253,258)$ & 1.6 & 102.5 \\
Parallel linear & $(255,255)$ & $(250,255)$ & 3.1 & 232.1 \\
\hline
\end{tabular}




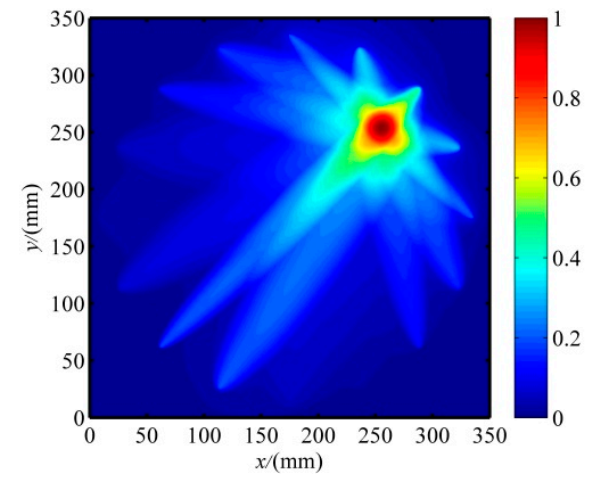

(a) Tomography image with a circular array

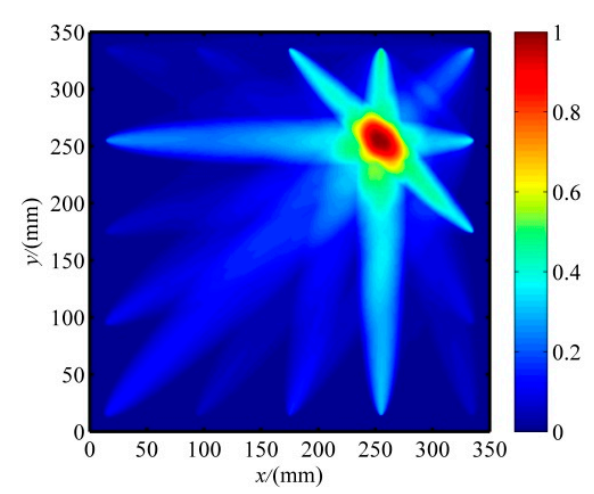

(c) Tomography image with a square array

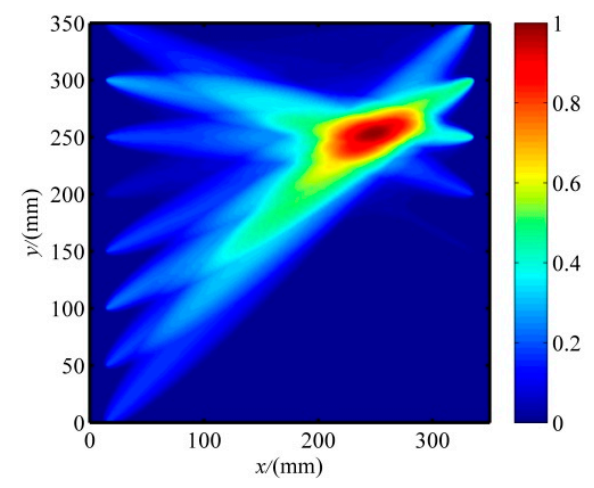

(e) Tomography image with a parallel linear array

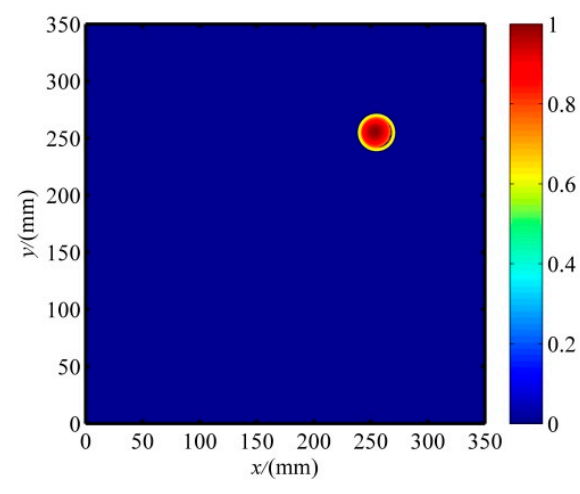

(b) Image with a circular array after a threshold value

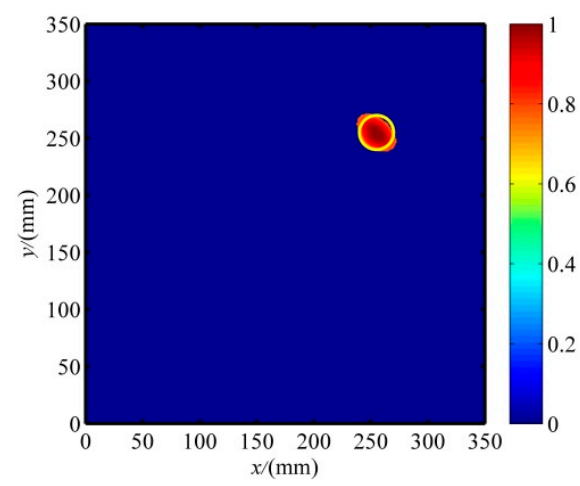

(d) Image with a square array after a threshold value

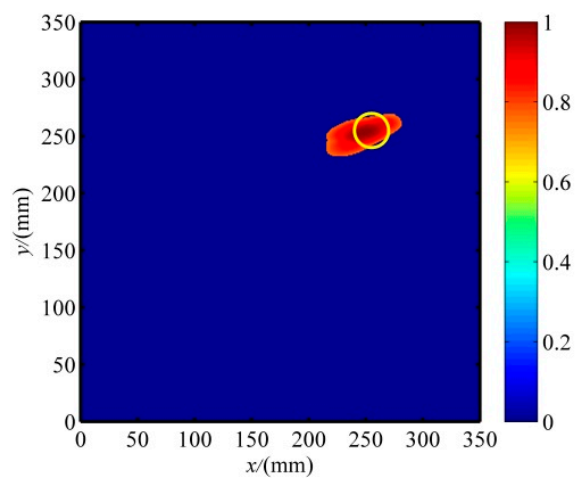

(f) Image with a parallel linear array after a threshold value

Figure 6. Comparison of the tomography images with three PZT sensor array configurations for a specimen with a hole.

\subsection{The Improved RAPID Algorithm Based on the Evaluation of Crack Orientation}

The reconstruction images of the crack in the specimen with three patterns of PZT sensor array configurations are shown in Figure 7, and the resultant location and damage shape are listed in Table 2. The tomography images had poor shape accuracies for all the sensor array configurations, but the predicted crack location was very close to the real location. This is because of the small width of the crack, the near lack of scattering along the crack orientation when the incident wave is parallel to the crack orientation, and SDC values close to 0 . Consequently, the crack orientation was not visible in the tomography images. The reconstruction images of three PZT sensor array configurations are 
not suitable for non-circular damage detection. Fortunately, the predicted location can be used to determine the crack orientation, as discussed in the following.

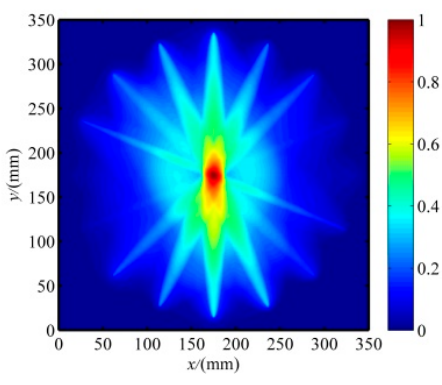

(a)

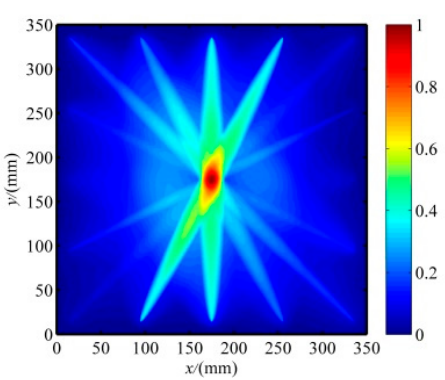

(b)

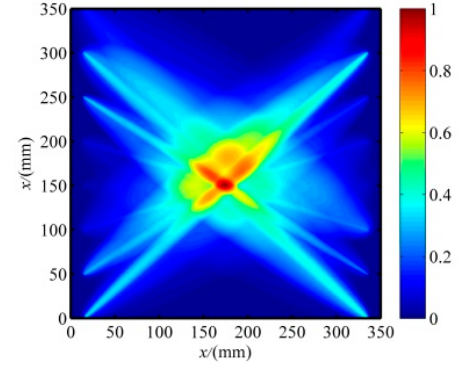

(c)

Figure 7. The initial reconstruction image of a crack without considering the crack orientation. (a) The image with a circular array. (b) The image with a square array. (c) The image with a parallel linear array.

Table 2. The resultant imaging accuracy of a crack without considering the crack orientation.

\begin{tabular}{cccc}
\hline Pattern of the PZT Sensor Array & Real Location & Predicted Location & Damage Shape \\
\hline Circular & $(175,175)$ & $(176,176)$ & Ellipse \\
Square & $(175,175)$ & $(176,176)$ & Oblique ellipse \\
Parallel linear & $(175,150)$ & $(175,151)$ & Elongated shape \\
\hline
\end{tabular}

The angular scattering patterns of the Lamb wave mode of cracks under different incident wave directions are well detailed in [23-28], which mostly focus on the transmission and reflection coefficients. This paper focuses on the SDC for tomography images. The commercial FEM software ANSYS was used for analyzing the angular scattering pattern of the SDC. SHELL181 elements were used to model the aluminum plate specimen. The mesh size of the finite element model was $1 \mathrm{~mm}$ which is smaller than one-twentieth of the Lamb wavelength at $30 \mathrm{kHz}$ to ensure the accuracy of the analysis results. The time step was set to $1 \mu \mathrm{s}$. Both the mesh size and the time step satisfy the criteria of transient dynamic analysis [38]. A total of 1,000,000 elements were used. Different incident Lamb wave directions were considered, i.e., $0^{\circ}, 22.5^{\circ}, 45^{\circ}, 67.5^{\circ}$, and $90^{\circ}$. The simulated scattered field of the crack in the specimen when the transmission Lamb wave passing the PZT sensors under an oblique incident wave direction of $45^{\circ}$ is plotted in Figure $8 \mathrm{a}$. A shadow effect appears behind the incident waves and the scatted waves from the crack. In addition, strong amplitude variations can be seen in certain areas. The signal measurements are made on the transmission area with a radius of $175 \mathrm{~mm}$ every $5^{\circ}$. The calculated SDC values under different incident wave directions are plotted in Figure 6b.

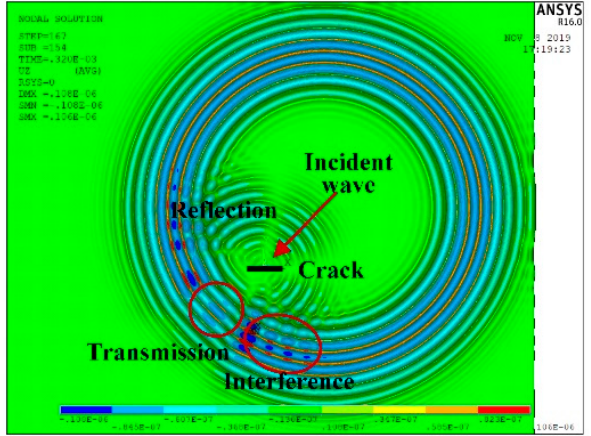

(a)

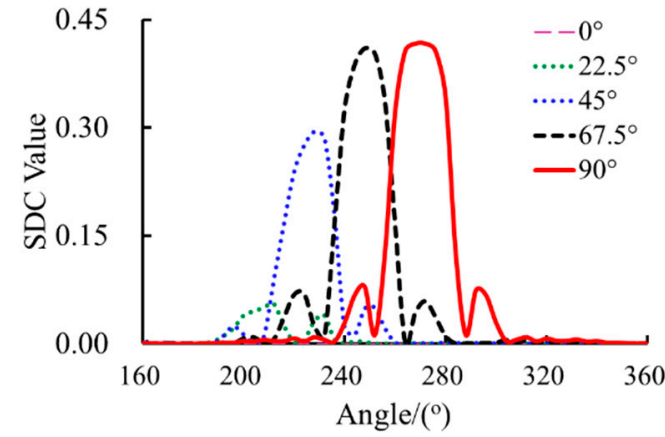

(b)

Figure 8. FEA results and signal difference coefficient (SDC) distributions: (a) displacement magnitude at oblique incidence $\left(45^{\circ}\right)$ and (b) SDC distributions with different incident wave directions. 
It can be found from Figure $8 \mathrm{~b}$ that the SDC curves are asymmetric about the normal direction of the crack orientation except for the normal incident wave (90); the SDC variation is substantially increased as the incident wave direction angle increases from $0^{\circ}$ to $67.5^{\circ}$ and then slowly increases from $67.5^{\circ}$ to $90^{\circ}$, which is not an excessively large angle to the normal direction of the crack. Such complicated directionality patterns of the SDC variation cannot be directly used to determine the crack orientation. Note that the SDC values of all sensing paths in the group of the incident wave direction of $0^{\circ}$ were much smaller than others, almost close to 0 . Thus, the actuator excited minimum SDC variations of all sensing paths in their own groups should be located in the extending line of the crack orientation. This is the criterion to find the first point of the line of the crack orientation; the other point is obtained by the initial reconstruction image of the crack, as presented in Figure 7. Therefore, an improved RAPID algorithm is proposed based on the evaluation of crack orientation, as described as follows.

Step 1: Find the actuator for which the SDC values of all the sensing paths in their own groups exhibit the minimum variation by experimental test. As for the circular array, the location of $A_{1}$ is the first point, as shown in Figure 9.

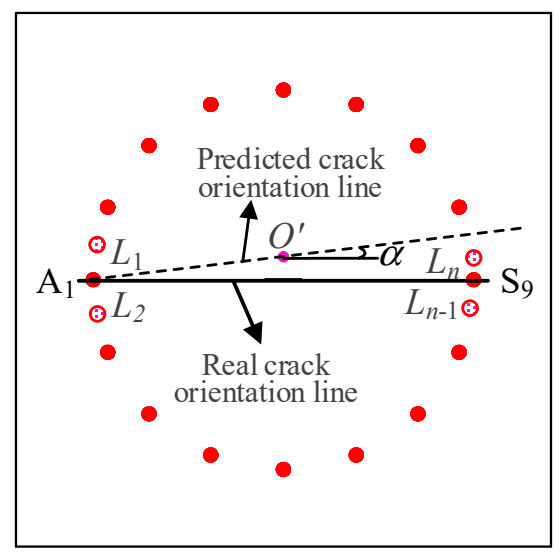

Figure 9. Determination of the crack orientation.

Step 2: Extract the center point $O^{\prime}$ of the initial reconstruction image (from Figure 7), thus the line between the points of $A_{1}$ and $O^{\prime}$ is the predicted crack orientation. The angle $\alpha$ between the predicted crack orientation line and the $x$ axis is used to describe the crack orientation. As for the circular array, actuator $A_{1}$ and $A_{9}$ both result in the minimum SDC variations of all the sensing paths in their own group.

Step 3: Define an additional SDC with a value of 1 for the predicted line of crack orientation, and reconstruct the crack image based on the initial reconstruction image in Figure 7.

The accuracy of the evaluated crack orientation depends on the density of the PZT sensor array. In applications, more sensors, $L_{1}, L_{2}, \ldots L_{\mathrm{n}}$, can be rearranged in the adjacent area of sensor $A_{1}$, as shown in Figure 9, to provide a more exact crack orientation. Based on the improved RAPID algorithm, the reconstruction tomography image is shown in Figure 10. The SDC modification is similar to the method in [34]. The difference is that the information of the initial reconstruction image is used in this paper. The reason is that the minimum SDC peak value, defined in [34], of the sensing path in its own group of the pre-determined actuator should not align with the line of crack orientation. 


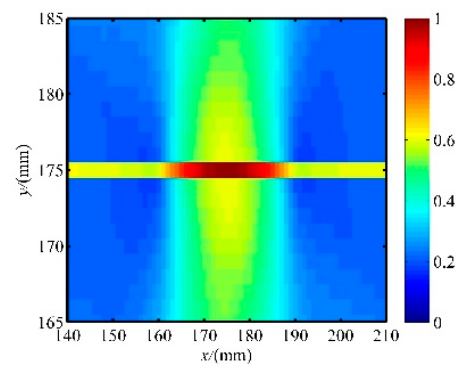

(a)

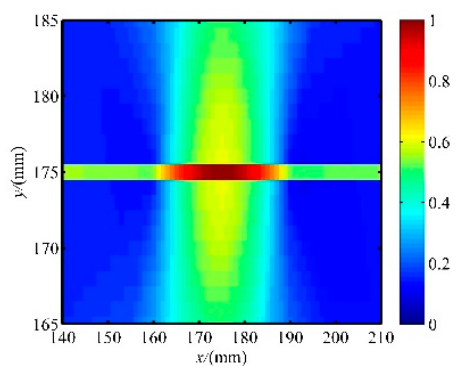

(b)

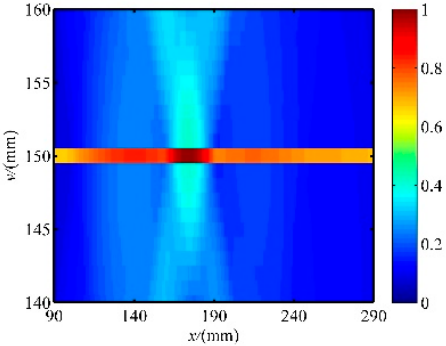

(c)

Figure 10. The improved RAPID algorithm with the predicted crack orientation: (a) image with a circular array, (b) image with a square array, and (c) image with a parallel linear array.

\subsection{Influence of PZT Sensor Arrays on the Images for the Crack Damage}

According to the improved RAPID algorithm, the reconstruction images of the crack in the specimen with three patterns of the PZT sensor array configuration are shown in Figure 11, and the resultant crack length errors are listed in Table 3. The circular and square array shows equivalent accuracy of crack length, which is better than the results of the parallel linear array. The crack orientation error is related to the density of the PZT array. As discussed in [6], the spatial resolutions of three sensor configurations are compared. Similarly, the angle resolution of the circular array is better than that of the square array; the parallel linear array is the best because of the higher density of the sensor on each edge line but may fail to predict the crack orientation when no sensors are allocated along the crack orientation direction. In summary, the circular array configuration is preferred for both hole and crack damage detection with the RAPID algorithm.

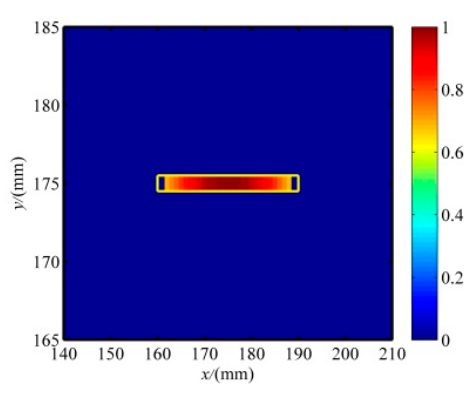

(a)

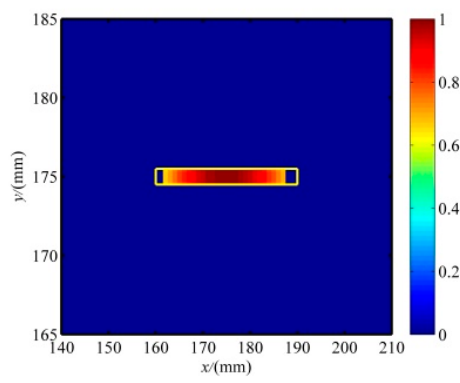

(b)

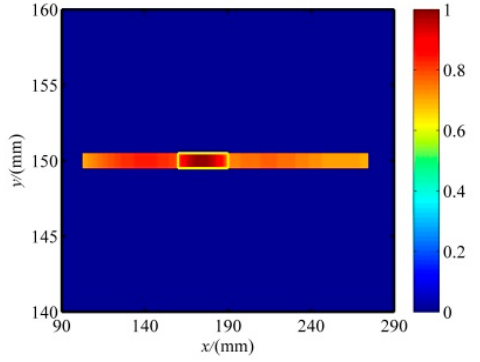

(c)

Figure 11. The improved images after SDC modification with three PZT sensor array configurations after a threshold value (a) The image with a circular array (b) The image with a square array (c) The image with a parallel linear array.

Table 3. Evaluation of crack length with three PZT sensor array configurations.

\begin{tabular}{cccc}
\hline Configuration Type & Real Crack Length & Predicted Crack Length & Length Error/\% \\
\hline Circular & 30 & 27 & 10.0 \\
Square & 30 & 26.1 & 13.0 \\
Parallel linear & 30 & 172.2 & 474 \\
\hline
\end{tabular}

\section{Conclusions}

This paper compares the reconstruction image qualities with three commonly used patterns of the PZT sensor array configurations, i.e., the circular, square, and parallel linear array configuration. The complex angular scattering pattern of the SDC distribution of the Lamb wave scattered from a crack is studied by ANSYS simulation. An improved RAPID algorithm is proposed by defining an additional SDC value of 1 in the line of the prediction crack orientation, which is determined by the point of the 
actuator where SDC values of all the sensing paths in its own group have the minimum variation and the center point of the initial tomography image. The effectiveness of the proposed method is verified. Reconstruction image qualities with three patterns of PZT sensor array configuration for both holes and cracks are compared. The results show that the circular array configuration is preferred for both hole and crack damage detection. The circular sensor array presents a better hole shape and size than the others and equivalent accuracy of crack length with the square sensor array but a higher angle resolution of crack orientation than the square sensor array. The square sensor array shows better damage quality than the parallel linear sensor array, but the latter may fail to estimate the crack orientation when no sensors are allocated along the crack orientation direction.

Author Contributions: S.W. was in contributed the methodology, experimental test, and writing-original. W.W. supervised this research and helped in modification. Y.S. was responsible for the data analysis, and the writing-review \& editing. Y.L. and S.J. were responsible for the simulation, and validation. All authors have read and agreed to the published version of the manuscript.

Funding: This research was funded by the National Natural Science Foundation of China (No. 51805161), Hunan Provincial Key Research and Development Program (No. 2017WK2051 and 2018GK2044), and the Hunan Provincial Natural Science Foundation (No. 2017JJ2084 and 2018JJ4084).

Acknowledgments: The work also benefited from the assistance of M.X. and B.L.

Conflicts of Interest: The authors declare no conflict of interest.

Data Availability Statement: The data and the MATLAB programs used to support the findings of this study are available from the corresponding author upon request.

\section{References}

1. Qing, X.; Li, W.; Wang, Y.; Sun, H. Piezoelectric Transducer-Based Structural Health Monitoring for Aircraft Applications. Sensors 2019, 19, 545. [CrossRef] [PubMed]

2. Li, X.; Yang, Z.; Chen, X. Quantitative damage detection and sparse sensor array optimization of carbon fiber reinforced resin composite laminates for wind turbine blade structural health monitoring. Sensors 2014, 14, 7312-7331. [CrossRef] [PubMed]

3. Khairi, M.T.M.; Ibrahim, S.; Yunus, M.A.M.; Faramarzi, M.; Sean, G.P.; Pusppanathan, J.; Abid, A. Ultrasound computed tomography for material inspection: Principles, design and applications. Measurement 2019, 146, 490-523. [CrossRef]

4. Su, C.; Jiang, M.; Liang, J.; Tian, A.; Sun, L.; Zhang, L.; Zhang, F.; Sui, Q. Damage Localization of Composites Based on Difference Signal and Lamb Wave Tomography. Materials 2020, 13, 218. [CrossRef] [PubMed]

5. Lambinet, F.; Sharif Khodaei, Z.; Aliabadi, F.M.H. Effectiveness of RAPID and SSM Algorithms on Composite Scarf Repair. Key Eng. Mater. 2018, 774, 535-540.

6. Zhao, X.; Royer, R.L.; Owens, S.E.; Rose, J.L. Ultrasonic Lamb wave tomography in structural health monitoring. Smart Mater. Struct. 2011, 20, 105002. [CrossRef]

7. Wang, D.; Ye, L.; Su, Z.; Lu, Y.; Li, F.; Meng, G. Probabilistic damage identification based on correlation analysis using guided wave signals in aluminum plates. Struct. Health Monit. 2010, 9, 133-144. [CrossRef]

8. Liu, Z.; Zhong, X.; Dong, T.; He, C.; Wu, B. Delamination detection in composite plates by synthesizing time-reversed Lamb waves and a modified damage imaging algorithm based on RAPID. Struct. Control Health Monit. 2017, 24, e1919. [CrossRef]

9. Sheen, B.; Cho, Y. A study on quantitative lamb wave tomogram via modified RAPID algorithm with shape factor optimization. Int. J. Precis. Eng. Man. 2012, 13, 671-677. [CrossRef]

10. Lee, J.; Sheen, B.; Cho, Y. Multi-defect tomographic imaging with a variable shape factor for the RAPID algorithm. J. Visual-Japan 2016, 19, 393-402. [CrossRef]

11. Zhao, J.; Miao, X.; Li, F.; Li, H. Probabilistic Diagnostic Algorithm-Based Damage Detection for Plates with Non-uniform Sections Using the Improved Weight Function. J. Vib. Eng. Technol. 2018, 6, 249-260. [CrossRef]

12. Li, W.; Xu, Y.; Qing, X.; Yang, Z. Quantitative imaging of surface cracks in polymer bonded explosives by surface wave tomographic approach. Polym. Test. 2019, 74, 63-71. [CrossRef] 
13. Croxford, A.J.; Wilcox, P.D.; Drinkwater, B.W. Quantification of sensor geometry performance for guided wave SHM. SPIE 2009, 7295, 72951H.

14. Hall, J.S.; Michaels, J.E. Analysis of distributed sparse array configurations for guided wave imaging applications. AIP Conf. Proc. 2011, 1335, 859-866.

15. Chen, X.; Michaels, J.E.; Michaels, T.E. Design of distributed sparse arrays for Lamb wave SHM based upon estimated scattering matrices. AIP Conf. Proc. 2014, 1581, 248-255.

16. Wu, Z.; Liu, K.; Wang, Y.; Zheng, Y. Validation and evaluation of damage identification using probability-based diagnostic imaging on a stiffened composite panel. J. Intell. Mater. Syst. Struct. 2015, 26, 2181-2195. [CrossRef]

17. Dziendzikowski, M.; Dragan, K.; Katunin, A. Localizing impact damage of composite structures with modified RAPID algorithm and non-circular PZT arrays. Arch. Civ. Mech. Eng. 2017, 17, 178-187. [CrossRef]

18. Guo, H.Y.; Zhang, L.; Zhang, L.L.; Zhou, J.X. Optimal placement of sensors for structural health monitoring using improved genetic algorithms. Smart Mater. Struct. 2004, 13, 528. [CrossRef]

19. Gao, H.; Rose, J. Ultrasonic sensor placement optimization in structural health monitoring using evolutionary strategy. AIP Conf. Proc. 2006, 820, 1687-1693.

20. Salmanpour, M.; Sharif Khodaei, Z.; Aliabadi, M. Transducer placement optimisation scheme for a delay and sum damage detection algorithm. Struct. Control Health Monit. 2017, 24, 1-20. [CrossRef]

21. Ismail, Z.; Mustapha, S.; Fakih, M.A.; Tarhini, H. Sensor placement optimization on complex and large metallic and composite structures. Struct. Health Monit. 2020, 19, 262-280. [CrossRef]

22. Fromme, P.; Sayir, M.B. Measurement of the scattering of a Lamb wave by a through hole in a plate. J. Acoust. Soc. Am. 2002, 111, 1165-1170. [CrossRef] [PubMed]

23. Liu, H.; Chen, X.; Michaels, J.E.; Michaels, T.E.; He, C. Incremental scattering of the A0 Lamb wave mode from a notch emanating from a through-hole. Ultrasonics 2019, 91, 220-230. [CrossRef] [PubMed]

24. Cai, Z. Study on the orientation detection of surface cracks by electromagnetic acoustic emission. Int. J. Distrib. Sens. Netw. 2019, 15, 1-11. [CrossRef]

25. Yeasin Bhuiyan, M.; Shen, Y.; Giurgiutiu, V. Interaction of Lamb waves with rivet hole cracks from multiple directions. Proc. Inst. Mech. Eng. C J. Mech. Eng. Sci. 2017, 231, 2974-2987. [CrossRef]

26. Fromme, P. Influence of guided ultrasonic wave scattering directionality on the detection sensitivity for SHM of fatigue cracks. Proc. SPIE 2010, 7650, 76501M.

27. Rajagopal, P.; Lowe, M.J.S. Angular influence on the scattering of fundamental shear horizontal guided waves by a through-thickness crack in an isotropic plate. J. Acoust. Soc. Am. 2008, 124, 2021-2030. [CrossRef]

28. Lu, Y.; Ye, L.; Su, Z.; Huang, N. Quantitative evaluation of crack orientation in aluminium plates based on Lamb waves. Smart Mater. Struct. 2007, 16, 1907-1914. [CrossRef]

29. Wang, Q.; Xu, Y.; Su, Z.; Cao, M.; Yue, D. An Enhanced Time-Reversal Imaging Algorithm-Driven Sparse Linear Array for Progressive and Quantitative Monitoring of Cracks. IEEE Trans. Instrum. Meas. 2018, 9456, 1-13. [CrossRef]

30. Yu, L.; Leckey, C.A. Lamb wave-based quantitative crack detection using a focusing array algorithm. J. Intell. Mater. Syst. Struct. 2013, 24, 1138-1152. [CrossRef]

31. He, J.; Guan, X.; Peng, T.; Liu, Y.; Saxena, A.; Celaya, J.; Goebel, K. A multi-feature integration method for fatigue crack detection and crack length estimation in riveted lap joints using Lamb waves. Smart Mater. Struct. 2013, 22, 105007. [CrossRef]

32. Zhou, C.; Su, Z.; Cheng, L. Quantitative evaluation of orientation-specific damage using elastic waves and probability-based diagnostic imaging. Mech. Syst. Signal Process. 2011, 25, 2135-2156. [CrossRef]

33. Tua, P.S.; Quek, S.T.; Wang, Q. Detection of cracks in plates using piezo-actuated Lamb waves. Smart Mater. Struct. 2004, 13, 643-660. [CrossRef]

34. Wang, Q.; Xu, J.; Wang, M.; Song, C. Lamb wave tomography technique for crack damage detection. Mech. Eng. J. 2016, 52, 30-36. [CrossRef]

35. Wang, Q.; Wang, M.; Yue, D.; Su, Z. A Lamb Wave-based Crack Diagnosis Method Using an Improved RAPID Algorithm. In Proceedings of the 8th European Workshop On Structure Health Monitoring, Bilbao, Spain, 5-8 July 2016.

36. Giurgiutiu, V. Tuned Lamb Wave Excitation and Detection with Piezoelectric Wafer Active Sensors for Structural Health Monitoring. J. Intell. Mater. Syst. Struct. 2005, 16, 291-305. [CrossRef] 
37. Lin, B.; Giurgiutiu, V. Modeling and testing of PZT and PVDF piezoelectric wafer active sensors. Smart Mater. Struct. 2006, 15, 1085-1093. [CrossRef]

38. Shen, Y.; Giurgiutiu, V. Combined analytical FEM approach for efficient simulation of Lamb wave damage detection. Ultrasonics 2016, 69, 116-128. [CrossRef]

(C) 2020 by the authors. Licensee MDPI, Basel, Switzerland. This article is an open access article distributed under the terms and conditions of the Creative Commons Attribution (CC BY) license (http://creativecommons.org/licenses/by/4.0/). 